\title{
Quantum Cryptographic Network based on Quantum Memories
}

\author{
Eli Biham \\ Computer Science Department \\ Technion \\ Haifa 32000, Israel \\ Bruno Huttner \\ Group of Applied Physics \\ University of Geneva \\ CH-1211, Geneva 4, Switzerland \\ Tal Mor \\ Department of Physics \\ Technion \\ Haifa 32000, Israel
}

(3.Mar.1996)

\begin{abstract}
Quantum correlations between two particles show non-classical properties which can be used for providing secure transmission of information. We present a quantum cryptographic system, in which users store particles in quantum memories kept in a transmission center. Correlations between the particles stored by two users are created upon request by projecting their product state onto a fully entangled state. Our system allows for secure communication between any pair of users who have particles in the same center. Unlike other quantum cryptographic systems, it can work without quantum channels and is suitable for building a quantum cryptographic network. We also present a modified system with many centers.
\end{abstract}

Typeset using REVTEX 


\section{INTRODUCTION AND MOTIVATION}

The main goal of cryptography, the secure transmission of messages, can be achieved using a secret key known only to the sender, Alice, and the receiver, Bob. The only known way which might allow two users to create an unconditionally secret key without sharing any common information in advance is quantum cryptography [1] 6]. In quantum cryptographic schemes Alice uses non-orthogonal quantum states (transmitted through a quantum channel) to transfer the key to Bob. Such states cannot be cloned hence any attempt by an eavesdropper, known as Eve, to get information on the key disturbs the transmitted signals and induces noise. This noise will be detected by Alice and Bob during the second stage of the transmission, which includes discussion over a public channel. The alternative to quantum key distribution schemes, Public Key Cryptography [7],8], relies on computational complexity assumptions such as the difficulty of factoring. To date, none of the existing public key cryptosystems is proven secure, even against attacker with limited computation power. Moreover, it was recently shown [9] that these complexity assumptions may not hold for a quantum computer (for example, a quantum computer should enable fast factorization). This implies that many public key cryptosystems, such as RSA [8], may be broken by quantum computers.

These new developments enhanced the interest in quantum cryptography and started a wide surge of interest in the field of quantum computing. However, building such computing devices is a difficult task, and quantum computing (which was invented a decade ago [10]) is only doing its first experimental steps. The building blocks of future quantum computers are one-bit and two-bit quantum logical gates [1] 14, which are currently under intensive development [15 17]. Building quantum computing devices to factor large numbers does not seem to be practical in the foreseeable future since it requires combining many one-bit and two-bit gates. However, a single two-bit gate also have intriguing uses in information processing and quantum communication such as teleporting a quantum state [18], and dense coding in quantum cryptography [6]. We shall show in this paper that the use of quantum gates together with a quantum memory (in which a quantum state can be maintained for a long time without loss of coherence) opens new directions in quantum cryptography. Our system may be practical long before quantum computers are, hence provides a short-term application for quantum gates.

One of the main disadvantages of quantum cryptography is its restriction to relatively short channels. This is due to the fact that, in contrast to classical channels, a quantum channel cannot use repeaters to amplify the signal without loss of coherence. Currently,

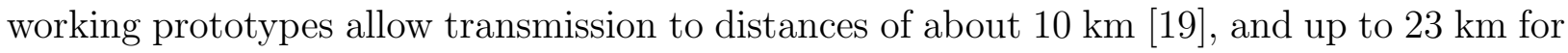
a recent experiment using installed telecom fibers [20]. Commercial systems may become available in the near future [21], so that two users will be able to communicate securely (if they are not too far). However, building quantum cryptographic networks based on the existing schemes円 seems to cause severe difficulties (which may even make it impractical):

\footnotetext{
${ }^{1}$ For a suggestion of a quantum cryptographic networks based on the existing schemes see [22].
} 
1. Quantum communication requires any pair of users to have a common quantum channel, or alternatively a center (or a telephone-like switching network) connected by quantum channels to all the users, which should match any pair of channels upon request; enhancing the security of the current world-wide telephone network (which contains about $N \approx 10^{9}$ users (telephones) ) using quantum cryptography requires huge investments in quantum channels and devices.

2. Any user must have the financial and technological abilities to operate complicated quantum devices.

3. The keys must be transmitted online, or else one would need to transmit $O\left(N^{2}\right)$ keys in advance to enable any pairs of users to communicate in secrecy.

4. The network must assure authenticity of the users.

It is important to have quantum cryptographic networks not suffering from these problems.

In this work we suggest a new cryptographic scheme in which users store quantum states in quantum memories, kept in a transmission center. Upon request from two users, the center uses two-bit gates to project the product state of two non correlated particles (one from each user) onto a fully entangled state. As a result, the two users can share a secret bit, which is unknown even to the center. Our scheme can operate without quantum channels, if the quantum states are "programmed" at the center. In that case, the scheme does not suffer from the four problems just mentioned, and can operate at any distance. Hence, it is especially appropriate for building a quantum cryptographic network of many users. Such a system actually shows some of the useful properties of the public key cryptosystems, but yet, it doesn't require computation assumptions.

In Section [1], we introduce our notation by reviewing various schemes for quantum cryptography and specifically describe the EPR-scheme. We then present a new two-party quantum cryptographic scheme which is a time-reversed EPR-scheme. In Section III, we present a quantum network based on the scheme presented in Section [1] with the addition of the quantum memories. In Section [V, we discuss the possibilities of implementing our scheme in practice. In Section $\nabla$, we present a more advanced network, based on quantum teleportation, where users can store their states in different centers and the centers teleport states upon request. This network uses quantum channels. However, it requires quantum channels only between the centers, so that the problems stated above do not arise. In Section $\nabla 1$ we summarize our results.

\section{A TIME-REVERSED EPR-SCHEME FOR QUANTUM CRYPTOGRAPHY}

Quantum cryptography provides techniques to distribute keys between two users, and its safety depends only on the fundamental rules of quantum mechanics. The legitimate users cannot prevent Eve from listening to their information exchange, but they will know if she does (hence, in this case, will not use this non-secret information). The first quantum cryptographic scheme, the Bennett Brassard (BB84) scheme [四], was presented already a decade ago. We describe it using the terminology of spin $1 / 2$ particles, but it can use 
any two-dimensional Hilbert space. A classical two-level system, such as a bistable device, can only be found in one of the two possible states, hence encodes one bit. In contrast, a quantum system can be prepared in any coherent superposition of the two basis states, which creates a much richer structure. Such a system is now known as a "qubit" 23] (i.e. quantum bit). For each qubit, Alice chooses at random whether to prepare her state along the $z$ or the $x$ axis, i.e., in one of the two eigenstates of either $\hat{S}_{z}$ or $\hat{S}_{x}$. This state, denoted by: $|\uparrow\rangle,|\downarrow\rangle,|\leftarrow\rangle$ or $|\rightarrow\rangle$ is then sent to Bob. It is agreed that the two states $|\uparrow\rangle$ and $|\leftarrow\rangle$ stand for bit value ' 0 ', and the other two states, $|\downarrow\rangle$ and $|\rightarrow\rangle$ stand for ' 1 '. Bob chooses, also at random, whether to measure $\hat{S}_{z}$ or $\hat{S}_{x}$. When his measurement is along the same axis as Alice's preparation (e.g. they both use $\hat{S}_{z}$ ), the measured value should be the same as hers, whereas when they use conjugate axes, there is no correlation between his result and Alice's original choice. In addition to the quantum channel, the legitimate users also use a classical channel which may be monitored, but cannot be modified by an eavesdropper (this assumption is discussed in [5], and is not required if Alice and Bob have a way to authenticate each other over the classical channel). By discussing over this channel Alice and Bob agree to discard all the instances where they did not use the same axes. The result should be two strings of perfectly correlated bits. As the choice of axis used by Alice is unknown to Eve, any interaction by her will unavoidably modify the transmission and introduce some errors. In practice however, the transmission will never be perfect and there will be some errors, even in the absence of an eavesdropper. Alice and Bob use the classical channel to compare some portion of their data and calculate the error rate. If it is not too high they can use classical information processing techniques, such as error correction and privacy amplification [5,24], to reduce the error rate to zero, while reducing the information obtained by Eve to zero as well. All these operations waste many bits (henceforth, $l$ ), so in order to be left with a key of $L$ bits Alice should send $L^{\prime}>2(L+l)$ qubits. A formal proof of security against an eavesdropper who is assumed to be limited only by the rules of quantum mechanics is still missing but may be available soon. These security aspects are widely discussed in the literature in case of the BB84 scheme but are common to all quantum cryptographic schemes and will not be discussed here.

More recently, another quantum key distribution scheme, based on EPR [25] correlations, was suggested by Ekert [2] and modified by Bennett, Brassard and Mermin [3]. We describe here the modified version which we call the EPR scheme. In this scheme Alice creates pairs of spin $1 / 2$ particles in the singlet state, and sends one particle from each pair to Bob. When the two particles are measured separately the results obtained for them are correlated. For example, if they are measured along the same axis, the results are opposite, regardless of the axis. Alice and Bob use the same sets of axes, say $\hat{S}_{z}$ and $\hat{S}_{x}$, and keep the results only when they used the same axis. It is noteworthy that, in the EPR scheme, the pairs could be created by any other party, including Eve herself.

As this point will prove crucial in our new scheme, let us discuss it in more details. The singlet state may be written in two ways:

$$
\begin{aligned}
\Psi^{(-)} & =\sqrt{\frac{1}{2}}\left(\left|\uparrow_{A \downarrow_{B}}\right\rangle-\left|\downarrow_{A} \uparrow_{B}\right\rangle\right) \\
& =\sqrt{\frac{1}{2}}\left(\left|\leftarrow_{A} \rightarrow_{B}\right\rangle-\left|\rightarrow_{A} \leftarrow_{B}\right\rangle\right),
\end{aligned}
$$


where the equality follows from $|\rightarrow\rangle=\frac{1}{2}(|\uparrow\rangle+|\downarrow\rangle)$ and $|\leftarrow\rangle=\frac{1}{2}(|\uparrow\rangle-|\downarrow\rangle)$, and where the subscripts 'A' and 'B', which stand for Alice and for Bob, can be omitted since we always write Alice's particle first. When Alice and Bob use the same axis, either $z$ or $x$, we use the first or the second equation respectively, to see that their measurements always yield opposite results. The singlet state is the only state which has that property. Therefore, as Alice and Bob may measure either of these two options, any deviation from the protocol by Eve (i.e. any attempt to create another state), will be detected with non zero probability. So Eve must create the required singlet state, from which she cannot extract any information about Alice's and Bob's measurement (see [2, 3] for more details).

The first aim of our paper is to suggest another scheme for quantum cryptography, which we shall call the time-reversed EPR-scheme. Let both Alice and Bob send one of the four states of BB84, | $\rangle,|\downarrow\rangle,|\leftarrow\rangle$ or $|\rightarrow\rangle$ to a third person whom we refer to as the center (the purpose of using this name shall be clarified in Section [I]). The center measures their qubits together to find whether or not the two particles are in a singlet state. This can be done by measuring the total-spin operator $\left(\hat{S}_{\text {total }}\right)^{2}$. If the result of the measurement is $s=0$, then the two particles are projected onto the singlet state. In that case Eq. 1 ensures that, if the two spins were prepared along the same axis, then they necessarily had opposite values (the projection of the states with identical spins on the singlet state is zero). As result, Bob knows Alice's bit and vice versa. However, from Eq. 1, a honest center, who followed the protocol and projected onto the singlet state, has absolutely no knowledge on these bits. For example, when Alice and Bob both used the vertical axis, the center does not know whether Alice had the up state and Bob the down state, or vice versa. If the measurement result is $s=1$, Alice and Bob cannot infer anything about the value of each other's bit, and shall discard the transmission. The probability of obtaining the singlet state is zero when Alice and Bob sent the same state (e.g., $\uparrow \uparrow)$, and is half in case they sent opposite states. Taking into account the case where Alice and Bob use different axes (which will also be discarded), we find that the overall probability to obtain a usable state is only one eighth.

To create a key with many bits, Alice and Bob send strings of quantum states $\left(L^{\prime}>\right.$ $8(L+l)$ qubits) to the center. The center must be able to keep them for a while (in case the states do not arrive at the same time from Alice and Bob), and then to measure the first pair, the second pair etc. The center tells Alice and Bob all cases in which the result of the measurement is a singlet, which happens in one fourth of the cases. Alice and Bob then compare their axes. When they used the same axis (which happens about half of the time), they know that their spins are necessarily opposite, and thus Bob can calculate Alice's bits to share a key with her. As in the BB84 scheme and the EPR schemes Alice and Bob use the classical discussion channel to estimate the error-rate. If it is tolerable they perform error correction and privacy amplification, to derive a final $L$-bit key.

The security of our protocol derives from the security of the EPR protocol, and relies on the fact that the singlet state is the only state for which the two spins are anticorrelated both in the $\hat{S}_{z}$ and in the $\hat{S}_{x}$ basis. However, as explained previously, if the center projects on the singlet state, he does not get any information on Alice's and Bob's bits. Therefore, a cheating center needs to project onto a different state (possibly entangled with his own system), which cannot give perfect anticorrelations along both $\hat{S}_{z}$ and $\hat{S}_{x}$ axes. Since the center cannot know in advance which basis was used by Alice and by Bob (the two density matrices corresponding to using $\hat{S}_{z}$ or $\hat{S}_{x}$ are identical), he will unavoidably introduce errors, 
which Alice and Bob shall identify during the discussion.

In fact, in terms of eavesdropping possibilities, our protocol and the EPR protocol are equivalent, as we show using the scheme presented in Fig. 1. In this scheme, two EPR pairs are created, one particle of each pair is sent to the center, and the second one to Alice and to Bob. In Fig. 1a, the center performs a measurement on his two particles first. A honest center, who follows the agreed protocol, projects the particles onto the singlet state. The two particles sent to Alice and to Bob are now in the singlet state as well. This is therefore equivalent to the EPR scheme. The only difference is that the projection onto the singlet state performed by the center succeeds with probability $1 / 4$ only. This means that the center will ask to discard $3 / 4$ of the transmission, but this does not affect the eavesdropping issue. A cheating center can send to Alice and Bob any state he wants, including any desired entanglement with his own system, by choosing an appropriate unitary transformation and the correct state on which to project his own particles. To show that, we start with the two singlet pairs and let the center introduce an ancilla in a state $A_{\text {init }}$. The state of the whole system is:

$$
\Phi_{A B C}=\frac{1}{2}(|\uparrow \downarrow\rangle-|\downarrow \uparrow\rangle) \otimes(|\uparrow \downarrow\rangle-|\downarrow \uparrow\rangle) \otimes A_{\text {init }} .
$$

The first particle of each singlet pair is sent to Alice and to Bob respectively, while the center keeps the second, together with his ancilla. The state $\Phi_{A B C}$ can thus be rearranged as:

$$
\Phi_{A B C}=\frac{1}{2}\left(|\uparrow \uparrow\rangle_{A B} \otimes|\downarrow \downarrow\rangle_{C}+|\downarrow \downarrow\rangle_{A B} \otimes|\uparrow \uparrow\rangle_{C}-|\uparrow \downarrow\rangle_{A B} \otimes|\downarrow \uparrow\rangle_{C}-|\downarrow \uparrow\rangle_{A B} \otimes|\uparrow \downarrow\rangle_{C}\right) \otimes A_{\text {init }},
$$

where the index $A B$ refers to the particles sent to Alice and Bob, and the index $C$ refers to the particles kept by the center. The center now applies a unitary transformation $U$ to entangle his particles with the ancilla in the following way:

$$
\begin{aligned}
U \Phi_{A B C}=\frac{1}{2}\left(|\uparrow \uparrow\rangle_{A B} \otimes|\downarrow \downarrow\rangle_{C} \otimes A_{1}+|\downarrow \downarrow\rangle_{A B} \otimes|\uparrow \uparrow\rangle_{C} \otimes A_{2}\right. \\
\left.-|\uparrow \downarrow\rangle_{A B} \otimes|\downarrow \uparrow\rangle_{C} \otimes A_{3}-|\downarrow \uparrow\rangle_{A B} \otimes|\uparrow \downarrow\rangle_{C} \otimes A_{4}\right),
\end{aligned}
$$

with $A_{i}$ any normalized states of the ancilla which are (in general) not orthogonal to one another. By projecting his state onto $\psi=\alpha|\downarrow \downarrow\rangle_{C}+\beta|\uparrow \uparrow\rangle_{C}-\gamma|\downarrow \uparrow\rangle_{C}-\delta|\uparrow \downarrow\rangle_{C} \quad$ (this projection succeeds with probability $1 / 4$ on average), the center creates the state

$$
\Psi_{A B C}=\frac{1}{2}\left(\alpha^{*}|\uparrow \uparrow\rangle_{A B} \otimes A_{1}+\beta^{*}|\downarrow \downarrow\rangle_{A B} \otimes A_{2}+\gamma^{*}|\uparrow \downarrow\rangle_{A B} \otimes A_{3}+\delta^{*}|\downarrow \uparrow\rangle_{A B} \otimes A_{4}\right),
$$

which is the most general state the center could create when cheating the EPR scheme [3]. This demonstrate the equivalence between Fig. 1a and the EPR scheme.

In Fig. 1b, the first measurement is performed by Alice and Bob, who project the particles onto the BB84 states. Therefore, the particles arriving at the center are also in the BB84 states, and this scheme is identical to ours. Since the relative time of the measurements cannot influence the outcome, all these schemes are equivalent. Following the same 
reasoning, but in two steps (first letting only Alice measure before the center) it is also possible to show that the security of the BB84 scheme implies the security of our scheme. Since the security of the EPR scheme implies the security of the BB84 scheme [3], our proof actually shows that the security of the three schemes is equivalent.

Using only the total spin measurement, less than one eighth of the qubits could be used. A better choice, although possibly more difficult to implement in practice, is to measure the Bell operator (defined in [26]) whose eigenstates (the Bell states) are the singlet state, $\Psi^{(-)}$ (equation 1), and the three other states:

$$
\begin{aligned}
& \Phi^{(+)}=\sqrt{\frac{1}{2}}(|\uparrow \uparrow\rangle+|\downarrow \downarrow\rangle)=\sqrt{\frac{1}{2}}(|\leftarrow \leftarrow\rangle+|\rightarrow \rightarrow\rangle), \\
& \Psi^{(+)}=\sqrt{\frac{1}{2}}(|\uparrow \downarrow\rangle+|\downarrow \uparrow\rangle)=-\sqrt{\frac{1}{2}}(|\leftarrow \leftarrow\rangle-|\rightarrow \rightarrow\rangle)
\end{aligned}
$$

and

$$
\left.\Phi^{(-)}=\sqrt{\frac{1}{2}}(|\uparrow \uparrow\rangle-\downarrow \downarrow\rangle\right)=\sqrt{\frac{1}{2}}(|\leftarrow \rightarrow\rangle+|\rightarrow \leftarrow\rangle),
$$

where the second expression for each of the Bell states is derived by expanding them (as was done for the singlet state) into the $(|\leftarrow\rangle,|\rightarrow\rangle)$ basis. Consider a case where Alice and Bob used the same basis. According to the result of the measurement, and to the choice of axes by Alice and Bob, their prepared states are known to be either correlated (e.g. if the result is $\Phi^{(-)}$and they both used the $z$ axis), or anticorrelated (e.g. if the result is still $\Phi^{(-)}$but they both used the $x$ axis).

The protocol goes as follows:

- The center retrieves the particles from Alice and Bob and measures the Bell operator on each pair. He gets one of the four above states, and tells his result to Alice and Bob.

- Alice and Bob tell each other the axis they used (but not the bit value). When they used different axes, they discard the transmission. Whenever they used the same axis, they know if their bits are correlated or anticorrelated. In this case half of the quantum states are used to derive the desired key, and $L^{\prime}>2(L+l)$ qubits are required.

The proof of security for this case is similar to the proof in the singlet case. A honest center, who projects the states onto the allowed states, cannot get any information on the bits. For example, if the center obtains the state $\Phi^{(+)}$, and Alice and Bob announce later that they used the horizontal axis, the center only knows that either both Alice and Bob have the left state, or both have the right state. But he cannot know which of these two possibilities occurred, hence has no information on the bit values. Moreover, similarly to the singlet case, $\Phi^{(+)}$is the only state for which Alice's and Bob's states have such correlations along both $x$ and $z$ axes. Therefore, a cheating center, who needs to create a different state in order to gain information, shall be detected with finite probability. 


\section{A QUANTUM CRYPTOGRAPHIC NETWORK}

In this Section we combine the reversed EPR scheme and the use of quantum memories into a classical network to present a quantum cryptographic network. The classical protocol for a network uses a "hidden file" managed by a communication center. Any user is allowed to put data (secret keys) in the file, under his name, but only the center has access to the data. Let there be $N$ users, and let each of them store many $L$-bits strings. Upon request from two users, the center uses their data and creates a secret key for them, which is shared by both of them: the center calculates the XOR of one string of the first user (say, $a_{1} \ldots a_{L}$ of Alice), and one string of the second user (say, $b_{1} \ldots b_{L}$ of Bob); the XOR of a string is calculated bit by bit using $c_{j}=a_{j} \oplus b_{j}$ (the parity of the two bits), and the resultant string, $C=c_{1} \ldots c_{L}$, is transmitted (via a classical unprotected channel) to Alice; Alice rederives Bob's string by calculating the XOR of her string with the received string, and can use Bob's string as their common key. Secure transmissions from each user to the center can be done either by personal delivery, trusted couriers or quantum key distribution. Such a classical key distribution scheme is perfectly secure if we assume that the center holding them is perfectly safe and trusted. No other person (except the center) can have any information on their key. Even a powerful eavesdropper who can impersonate the center and all the users cannot eavesdrop, since the center and each of the legitimate users can use some of the secret bits for authenticating each other. Alice and Bob need to trust the center for two different purposes:

1. To "forget" their secret key (and not trying to listen to the messages transmitted using that key);

2. To authenticate one to the other in case they have no other way of authentication. This is a new possibility of authentication, added to the two options, previously mentioned in Section [1]. Thus the assumption of having classical channels which cannot be modified can be completely removed, even if the users have no other way to authenticate each other.

The main reason why this simple scheme is not satisfactory in practice, is that it concentrates too much power in the distribution center. Indeed the center can understand all the secret communications going through its distribution web, or connect Alice to an adversary instead of to Bob. Even if we assume that the center is trusted, any eavesdropper who manages to get access to it could decipher all the communications.

Using a quantum memory instead of a classical memory is the key-point in deriving the quantum network, hence we present it in more details. While a classical bit can only represent a zero or a one, a qubit $|\phi\rangle=\alpha|0\rangle+\beta|1\rangle$ is described by two complex numbers (up to freedom of overall phase and normalization requirement). Unlike a classical memory which keeps $n$ classical independent bits, the $n$ qubits in a quantum memory can have non-classical correlations, and the state of a quantum register is described by $2^{n}$ complex numbers (up

to freedom of overall phase and normalization requirement). Even the simplest form of a memory (where the qubits are never correlated) is very important in quantum cryptography. For example, it allows doubling the efficiency of the BB84 scheme to use only $L^{\prime}>L+l$ qubits: instead of measuring the state sent by Alice immediately, Bob keeps it in a quantum 
memory; waits for Alice to disclose her basis; and then measures the state in the correct basis. In this case, the BB84 scheme can be used directly to transmit messages instead of random keys; Alice decide in advance which qubits will be used for error estimation, and encode the message using the rest of the qubits and using block-coding techniques to allow for error-correction. A quantum memory is also a basic tool for eavesdropping attacks, as it allows Eve to couple the transmitted states to an ancilla, and delay the measurement on the ancilla till the public exchange of basis.

We now present a quantum key distribution network which uses a quantum file instead of the classical hidden file, and removes the requirement of a trusted center. Alternatively, we can release the usual assumption of quantum cryptography [5] - that classical channels cannot be modified by Eve - if we are willing to trust the center for authentication (without trusting him for "forgetting" their qubits). Instead of storing $L$ classical bits to make a future key, each user shall store $L^{\prime}$ quantum states (qubits) in specially devised quantum memories kept in a center. Upon request from two users, the center performs the time reversed EPR scheme described in the previous Section and creates correlations between the bits. The resulting string $\mathrm{C}$, which holds the correlation data is sent to Alice via a classical channel. As in the classical case, using string C, Alice can calculate Bob's string to derive a final common key of $L$ bits. If Alice and Bob compare bases after deriving the data from the center, then, as explained in Section 【1, any attempt by the center to obtain the value of these bits will create errors and be discovered by Alice and Bob. The center therefore does not need to be trusted anymore. Unlike other quantum schemes, the actual (online) distribution of the secret keys is performed on classical channels. First, the center let Alice and Bob know the state he got. Then, Alice and Bob continue as in the other two schemes previously described to obtain the final key. All the quantum communication is done in advance, when the users "deposit" their quantum strings in the center (preferably in a personal meeting).

When $L$-bit strings are stored in a classical hidden file, two users derive $L$-bit strings of correlated bits. Using quantum states for representing the bits, longer strings of length $L^{\prime}>L+l$ are required, since some bits will be used for error estimation, error correction and privacy amplification. The exact ratio depends on the expected error-rate in the channel. Only the bits which are encoded in the same basis by both users can be used, therefore $L^{\prime}>2(L+l)$ bits are actually required (we assume that the more efficient scheme of measuring the Bell basis is used).

Let us summarize the protocol as follows:

- In the preparation step the user sends (gives) $L^{\prime}$-bit strings to the center, each bit is represented by one of the four states of the BB84 protocol. The center keeps these quantum states in a quantum file without measuring them. It is important that the system used for keeping the quantum states will preserve them for a long time (as long as required until the actual key distribution is performed).

- When Alice and Bob wish to obtain a common secret key, they ask the center to create correlations between two strings, one of Alice and one of Bob. The center performs the Bell operator measurement on each pair of photons, which projects them onto one of the Bell states, and tells Alice and Bob the result he obtained. After Alice gets the results from the center (and not before that), Alice and Bob compare the basis 
they used and keep only the bits for which they used the same basis. In this case, and according to the state obtained, the states of Alice and Bob are either correlated or anticorrelated. So, Alice for example inverts all her bits which should be anticorrelated with Bob's. The remaining string should be identical with Bob's, apart from possible errors.

- A honest center, who performed the correct projections on the Bell states does not get any information on the string.

- A cheating center (or any other eavesdropper who might have had access to the quantum files), who modified the allowed states, unavoidably introduced errors between the two strings.

- Alice and Bob perform error estimation, error correction and privacy amplification to derive a final key.

The quantum channel is used only as a preparation step between each user and the center, and all the online communication is done via a classical channel. Yet, only $O(N)$ keys are required to enable secret communication between any pair of the $N$ users. Any other quantum key distribution scheme requires $O\left(N^{2}\right)$ keys, or else requires online quantum communication. In fact, our scheme does not require quantum channels at all. As in old implementations of quantum cryptography [27], the four quantum states can be chosen in any 2-dimensional Hilbert space. Instead of sending them, each user could arrive once in a while to the center, and "program" his states into the quantum file. If the memory can keep the states unperturbed long enough then each user can put as many strings as he needs till his next visit to the center. By using personal delivery of the quantum states we replace the distance limitation of all other schemes by a time limit, and solve the problems of a quantum cryptographic network which were described in the introduction. All the technically involved steps, such as storing qubits and performing Bell measurements occur only at the center.

\section{IMPLEMENTATIONS}

Our scheme requires the possibility to program, store and manipulate quantum bits rather than to transmit them. Therefore any 2-dimensional Hilbert space system can be considered and this opens a variety of possible implementations. Fortunately, almost the same requirements appeared recently in quantum computing, and are being thoroughly investigated by both theorists [11 14], and experimentalists [15 17]. The main difference in the requirements is that the quantum bits in our scheme are subjected only once to a unitary operation of calculation hence the problem of decoherence is much less severe.

We estimate ${ }^{2}$ that it may be possible to implement a working prototype of our scheme within a few years, with small modifications of existing technology. Such a prototype shall be able to keep quantum states for a few minutes and to allow to perform two-bit operations

\footnotetext{
${ }^{2}$ The following suggestions were investigated with the help of D. DiVincenzo [28].
} 
on them. At the moment, the best candidates for combining these two operations are ion traps. In ion traps the quantum bits can be kept in internal degrees of freedom (say, spin) of the ions, and in phononic degrees of freedom of few ions together. It is already possible to keep quantum states in the spin of the ions for more than 10 minutes [29] and in principle it is possible to keep them for years. These ion traps are also the among the best candidates for two-bit operations since there are ways to use the phononic degrees of freedom to perform two-bit operations 14. Barenco et al. [13 realized that a single quantum controlled-NOT logical gate would be sufficient to perform the Bell measurement. Using ion traps it is possible to (partially) perform the Bell measurement as shown both in theory [14 and in an experiment [17]. Combining together the two experiments to have both long lived quantum states and the possibility to manipulate them will allow two users to derive a few secure common bits.

The way to establish a real working scheme with a center and with many users is still long. The main obstacle is that it is currently impossible to transfer a quantum state from one ion trap to another. A real network should allow each user to program his quantum states in a register (say, at least one separated ion trap for each user). Then, upon request of two users, the center should be able to move one bit of each of the two users to another ion trap where he can perform the Bell measurement without disturbing the other quantum states. Recently, the possibility of doing this arose from the idea of combining ion-traps (where the ions are well controlled) and QED-cavity together, and to use the same internal degrees of freedom for both 28,30. We shall call this combination cavitrap for convenience. In QED cavity [15] the internal degrees of freedom are coupled to photons and not to phonons. Recently, another group [16] has shown that it is possible to use polarization states of photons instead of using the $|0\rangle$ and $|1\rangle$ Fock state. If such photon states are used in a cavitrap, it may be possible to use them to transmit a quantum state from one cavitrap to another 28. In some sense, this will be an implementation of the nuclear spins based "quantum gearbox" suggested by DiVincenzo [12].

All this discussion would be nothing but a fantasy if our scheme would yield the only application of these ideas. However, as we already said, similar (and more complicated) ideas are required for other usages of quantum gates in both quantum computing and quantum information, and a lot of effort is invested in both the theory and application of quantum gates. Quantum memory is less discussed in quantum computing, but more in quantum cryptography, and the use of quantum memory to attack quantum cryptographic schemes is already appreciated. Nevertheless, the use of a quantum memory by the legitimate users of a quantum cryptographic scheme is not common, due to the desire to present protocols which may be implemented with existing technology. However, bypassing this "habit" and using a forthcoming tool is certainly justified when it allows to carry out new and important tasks which cannot be performed without it. The use of quantum memory in our network is clearly such a case, and we are sure that it will allow many other new tasks in the future. While we were working on this paper, it was suggested to use both quantum gates and quantum memory for purification of singlets [31], an idea which is useful for quantum cryptography as well. 


\section{WORLD-WIDE NETWORK OF MANY CENTERS}

The network of Section IIT is well suited for communication among users who are not far away and can arrive to the center. For two users who are far away and cannot come to the same center our network may not be appropriate. We now show that it can be modified to be useful also in this case. Let there be many centers, and many users in each center. Each two centers should share many EPR singlet pairs and upon request of users of the two centers, one center would teleport [18] the qubits of his user to the other center. This operation can be done with 100\% efficiency (but it may, however, increase the error-rate). The singlet pairs can be transmitted using any quantum cryptography scheme or even using a teleportation scheme and a supercenter who share EPR pairs with all centers. However, the transmission and distribution of singlet pairs require quantum channels even if done in advance".

Do we lose all the benefits we gained before? Certainly not. Still, only the centers need to have ability of performing quantum operations. All quantum transmission is done in advance, and yet, there is no need for $O\left(N^{2}\right)$ strings, since the number of centers is much smaller than the number of users. Authentication is still simple. One problem of any quantum channel is the limit on its length. Our first scheme (with one center) replaced it by a limit on time. It would be bad to have both problems in a network of many centers. However, the suggestion of purifying singlets [31] enables transmitting signals to longer distances. Our scheme can make an excellent use of it, since only the centers need to have the technological ability of purifying singlets. Moreover, several transmission stations can be put in between to improve transmission (this idea was suggested by DiVincenzo [28]) by performing purification of singlets between any two neighboring stations and then use teleportation from one station to the next to derive purified singlets shared by the centers.

\section{SUMMARY AND DISCUSSION}

In this paper we introduced a new scheme for quantum cryptography based on a timereversed EPR scheme. We suggested two new types of networks: a classical one based on hidden files, and one based on quantum files. The security of key distribution protocols in these networks does not rely on computational complexity assumptions. Both networks can be used to distribute keys in a secure way among any two users using simple online communication via classical channel. In the case of hidden files it is done with the help of a trusted center who can have access to all the information exchanged through its lines. Using quantum files, the center need not be trusted. Users have the means to check whether the center, or any other eavesdropper, tried to obtain information on the transmitted messages.

The one-center quantum network we suggest does not require any quantum channel at all, and can be implemented in a center where each user "programs" his states into a quantum

\footnotetext{
${ }^{3}$ And transporting quantum states to deliver them by a personal meeting is similar to transmitting them through channels
} 
memory. We estimate that a working prototype may be built in the near future using iontraps technology. A real network can be built when the problem of transmitting a quantum state from one trap to another is be solved (perhaps using cavitrap with polarization states). The machinery required for our scheme is also required for much more complicated tasks such as purification and quantum computing. In this respect, our scheme may represent a first practical application for these new devices, which are now being planned in various laboratories. We hope that our work will motivate more research for systems which can both keep a quantum state for a long time, and allow for the desired programming and measurements. We didn't pay much attention to the delicate problem of programming the states. The programming requires a simple equipment to make sure that the center does not eavesdrop on the preparation step. While cavitraps are very complicated, programming the polarization state of each of the photons may be quite simple in the future.

A future system of secure communication based on the protocol of Section $\square$ would involve a number of large transmission centers, which can exchange EPR correlated particles and store them, together with the qubits deposited at the center by various users. Secure communication between any pair of users would then imply teleportation of the states to the same center, followed by the creation of correlations between the two strings.

\section{ACKNOWLEDGMENTS}

We thank A. Peres for many discussions and we also thank C. H. Bennett, G. Brassard, C. Crépeau, A. Ekert and L. Vaidman for helpful remarks. Especially we would like to thank D. DiVincenzo for his great help in analyzing the possible implementations of our protocol. B.H. gratefully acknowledges financial support from the Swiss Fonds National de Recherche Scientifique. 


\section{REFERENCES}

[1] C. H. Bennett and G. Brassard, in Proceedings of IEEE International Conference on Computers, Systems and Signal Processing, Bangalore, India (IEEE, New York, 1984) p. 175.

[2] A. K. Ekert, Phys. Rev. Lett. 67, 661 (1991).

[3] C. H. Bennett, G. Brassard and N. D. Mermin, Phys. Rev. Lett. 68, 557 (1992).

[4] C. H. Bennett, Phys. Rev. Lett. 68, 3121 (1992).

[5] C. H. Bennett, F. Bessette, G. Brassard, L. Salvail, and J. Smolin, Journal of Cryptology 5, 3 (1992).

[6] C. H. Bennett and S. J. Wiesner Phys. Rev. Lett. 69, 2881 (1992).

[7] W. Diffie and M. E. Helman, IEEE Trans. Info. Theo. 22, 644 (1976).

[8] R. L. Rivest, A. Shamir and L. Adelman, Comm. ACM 21, 120 (1978).

[9] P. W. Shor, Proc. 35th Ann. Symp. on FOCS, IEEE Press, Los Alamos, CA (1994), 116.

[10] D. Deutsch Proc. R. Soc. Lond. A425 73 (1989).

[11] D. Deutsch Proc. R. Soc. Lond. A420 97 (1985).

[12] D. DiVincenzo, Phys. Rev. A51, 1015 (1995).

[13] A. Barenco, D. Deutsch, A. Ekert and R. Jozsa, Phys. Rev. Lett. 74, 4083 (1995).

[14] J. I. Cirac and P. Zoller Phys. Rev. Lett. 74, 4091 (1995).

[15] L. Davidovich, N. Zagury, M. Brune, J. M. Raimond and S. Haroche, Phys. Rev. A50, R895 (1994).

[16] Q. A. Turchette, C. J. Hood, W. Lange, H. Mabuchi and H. J. Kimble, Phys. Rev. Lett. 75, 4710 (1995).

[17] C. Monroe, D. M. Meekhof, B. E. King, W. M. Itano and D. J. Wineland, Phys. Rev. Lett. 75, 4714 (1995).

[18] C. H. Bennett, G. Brassard, C. Crépeau, R. Jozsa, A. Peres and W. K. Wootters, Phys. Rev. Lett. 70, 1895 (1993).

[19] P. D. Townsend, J. G. Rarity, and P. R. Tapster, Electronics Letters 29, 634 (1993); P. D. Townsend, Electronics Letters 30, 810 (1994).

[20] A. Muller, H. Zbinden and N. Gisin, Nature 378, 449 (1995).

[21] R. J. Hughes, D. M. Alde, P. Dyer, G. G. Luther, G. L. Morgan and M. Schauer, Contemporary Physics 36, 149 (1995).

[22] P. D. Townsend, S. J. D. Phoenix, K. J. Blow and S. M. Barnett, Electronics Letters 30, 1875 (1994).

[23] B. Schumacher, Phys. Rev A51, 2738 (1995).

[24] C. H. Bennett, G. Brassard, C. Crépeau and U. Maurer, IEEE Trans. Info. Theo. 41,1915 (1995).

[25] Einstein, A., Podolsky, B. and Rosen, N., Phys. Rev., vol. 47, 1935, pp. 777-780 (Reprinted in Quantum theory and measurement, J. A. Wheeler and W. Z. Zurek Eds, Princeton University Press, 1983).

[26] S. L. Braunstein, A. Mann and M. Revzen, Phys. Rev. Lett. 68, 3259 (1992).

[27] C. H. Bennett, G. Brassard, S. Breidbart and S. Wiesner, Advances in Cryptology: Proc. Crypto 82, Plenum Press, 267 (1982).

[28] D. DiVincenzo, private communication. 
[29] J. J. Bollinger, D. J. Heinzen, W. M. Itano, S. L. Gilbert and D. J. Wineland, IEEE Trans. Inst. and Meas. 40, 126 (1991).

[30] T. Pellizzary, S. A. Gardiner, J. I. Cirac and P. Zoller, Phys. Rev. Lett. 75 , 3788 (1995).

[31] C. H. Bennett, G. Brassard, S. Popescu, B. Schumacher, J.A. Smolin and W.K. Wootters, Phys. Rev. Lett. 76, 722 (1996). 


\section{FIGURES}

FIG. 1. Two processes, which we use to prove the security of the protocol. In both figures, one particle of each EPR correlated pair (denoted by dashed lines) is sent to the center, who performs a Bell measurement. We consider only the case where the result of the measurement is a singlet state. The second particles are sent to Alice and to Bob respectively, who project them onto the BB84 states. In a), the first measurement is done by the center. The particles arriving to Alice and Bob are therefore in the singlet state like in the EPR based protocol. In b), the first measurement is performed by Alice and Bob. Each particle sent to the center is therefore in one of the BB84 states. This is similar to our protocol. 